Supporting Information for the Paper

\title{
Lewis Acid-Promoted Intermolecular Carbonyl-Ene Reaction of Enantiopure 4-Oxoazetidine-2-carbaldehydes. Rapid Entry to Novel Fused Polycyclic $\beta$-Lactams
}

\author{
Benito Alcaide, ${ }^{* a}$ Pedro Almendros, ${ }^{b}$ Carmen Pardo, ${ }^{\mathrm{a}}$ Carolina Rodríguez-Ranera, ${ }^{\mathrm{a}}$ and Alberto \\ Rodríguez-Vicente ${ }^{\mathrm{a}}$ \\ a Departamento de Química Orgánica I. Facultad de Química. Universidad Complutense 28040- \\ Madrid. Spain \\ bInstituto de Química Orgánica General, CSIC, Juan de la Cierva 3, 28006-Madrid, Spain \\ E-mail: alcaideb@quim.ucm.es
}

\section{Table of Contents}

Preparation and Characterization of Homoallylic Alcohol Derivatives 2-4 $\quad$ S2

Preparation and Characterization of the $O$-Acetylmandelates of Alcohol (+)-2b $\quad$ S6

Preparation and Characterization of Methanesulfonates (+)-5b and (+)-5b $\quad$ S7

Preparation and Characterization of Diels-Alder Cycloadduct (+)-6c $\quad$ S8

$\begin{array}{ll}\text { Preparation and Characterization of tricyclic } \beta \text {-lactam (+)-7a } & \text { S8 }\end{array}$

$\begin{array}{ll}\text { Preparation and Characterization of bicyclic amide ( } \pm \text { )-8b } & \text { S8 }\end{array}$

Preparation and Characterization of bicyclic $\beta$-lactam (+)-11b $\quad$ S9 
(3R,4S)-4-[(R)-1-Hydroxy-2-(1-cyclopentenyl)-ethyl]-3-methoxy-1-(p-methoxyphenyl)2-azetidinone, (+)-2a. From $100 \mathrm{mg}(0.43 \mathrm{mmol})$ of aldehyde (+)-1a, $88 \mathrm{mg}(65 \%)$ of compound (+)-2a was obtained as a colorless solid. Mp: $140-142{ }^{\circ} \mathrm{C}$ (hexanes/ethyl acetate). $[\alpha]_{\mathrm{D}}=+127.8(c$ 1.0, $\left.\mathrm{CHCl}_{3}\right) .{ }^{1} \mathrm{H}-\mathrm{NMR}: \delta 1.86(\mathrm{~m}, 2 \mathrm{H}), 2.31(\mathrm{~m}, 6 \mathrm{H}), 2.49(\mathrm{~d}, 1 \mathrm{H}, J=3.4 \mathrm{~Hz}), 3.69(\mathrm{~s}, 3 \mathrm{H}), 3.80(\mathrm{~s}$, $3 \mathrm{H}), 4.20(\mathrm{~m}, 1 \mathrm{H}), 4.27(\mathrm{t}, 1 \mathrm{H}, J=5.0 \mathrm{~Hz}), 4.63(\mathrm{~d}, 1 \mathrm{H}, J=5.1 \mathrm{~Hz}), 5.49(\mathrm{br} \mathrm{s}, 1 \mathrm{H}), 6.86$ and 7.44 (m, each 2H). ${ }^{13} \mathrm{C}-\mathrm{NMR}: \delta$ 165.1, 156.7, 140.3, 139.7, 126.9, 120.4, 114.1, 82.6, 69.1, 60.4, 59.6, 55.4, 35.6, 35.0, 32.5, 23.3. IR (KBr, cm-1): v 3408, 1720. MS (EI), m/z: $317\left(\mathrm{M}^{+}, 32\right), 87$ (100). Anal. Calcd for $\mathrm{C}_{18} \mathrm{H}_{23} \mathrm{NO}_{4}$ : C, 68.12; H, 7.30; N, 4.41. Found: C, 68.22; H, 7.33; N, 4.39.

$(3 R, 4 S)-4-[(R)-1-H y d r o x y-2-(1-c y c l o p e n t e n y l)]-1-(p-m e t h o x y p h e n y l)-3-p h e n o x y-2-$

azetidinone, (+)-2b. From $100 \mathrm{mg}(0.34 \mathrm{mmol})$ of aldehyde (+)-1b, $75 \mathrm{mg}(60 \%)$ of compound (+)-2b was obtained as a colorless solid. Mp: 149-151 ${ }^{\circ} \mathrm{C}$ (hexanes/ethyl acetate). $[\alpha]_{\mathrm{D}}=+157.5(c$ 1.0, $\left.\mathrm{CHCl}_{3}\right) .{ }^{1} \mathrm{H}-\mathrm{NMR}: \delta 1.91(\mathrm{~m}, 2 \mathrm{H}), 2.40(\mathrm{~m}, 7 \mathrm{H}), 3.81(\mathrm{~s}, 3 \mathrm{H}), 4.33(\mathrm{~m}, 1 \mathrm{H}), 4.66(\mathrm{t}, 1 \mathrm{H}, J=5.6$ Hz), 5.39 (d, 1H, J = 5.4 Hz), 5.54 (br s, 1H), 6.89 (m, 2H), $7.11(\mathrm{~m}, 3 \mathrm{H}), 7.35$ (m, 2H), 7.52 (m, 2H). 13C-NMR: $\delta$ 163.8, 157.4, 156.8, 140.0, 130.8, 129.7, 127.7, 122.7, 120.4, 115.9, 114.1, 79.6, 69.5, 60.9, 55.4, 35.9, 35.0, 32.5, 23.3. IR (KBr, cm-1): v 3529, 1743. MS (EI), $m / z: 380\left(\mathrm{M}^{+}+1,11\right)$, $379\left(\mathrm{M}^{+}, 39\right), 149$ (100). Anal. Calcd for $\mathrm{C}_{23} \mathrm{H}_{25} \mathrm{NO}_{4}$ : C, 72.80; H, 6.64; N, 3.69. Found: C, 72.93; H, 6.57; N, 3.72.

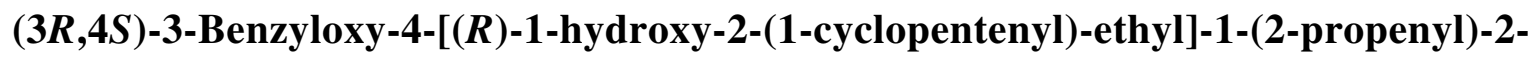

azetidinone, (+)-2c. From $100 \mathrm{mg}(0.41 \mathrm{mmol})$ of aldehyde (+)-1c, $81 \mathrm{mg}(60 \%)$ of compound (+)-2c was obtained as a colorless solid. Mp: 65-67 ${ }^{\circ} \mathrm{C}$ (hexanes/ethyl acetate). $[\alpha]_{\mathrm{D}}=+41.1$ (c 1.0, $\left.\mathrm{CHCl}_{3}\right) .{ }^{1} \mathrm{H}-\mathrm{NMR}: \delta 1.86(\mathrm{~m}, 2 \mathrm{H}), 2.34(\mathrm{~m}, 7 \mathrm{H}), 3.67(\mathrm{t}, 1 \mathrm{H}, J=5.0 \mathrm{~Hz}), 3.82(\mathrm{ddt}, 1 \mathrm{H}, J=15.6,5.1$, $1.6 \mathrm{~Hz}), 4.67(\mathrm{~d}, 1 \mathrm{H}, J=4.9 \mathrm{~Hz}), 4.70$ and $4.98(\mathrm{~d}$, each $1 \mathrm{H}, J=11.7 \mathrm{~Hz}), 5.24(\mathrm{~m}, 2 \mathrm{H}), 5.48$ (br s, 1H), $5.80(\mathrm{~m}, 1 \mathrm{H}), 7.35(\mathrm{~m}, 5 \mathrm{H}) .{ }^{13} \mathrm{C}-\mathrm{NMR}: \delta 167.6,140.1,136.8,132.0,128.5,128.1,127.9,127.4$, 118.3, 80.8, 73.1, 68.9, 60.5, 44.2, 35.9, 34.9, 32.4, 23.3. IR (KBr, $\left.\mathrm{cm}^{-1}\right): v$ 3427, 1747. MS (CI), $\mathrm{m} / z$ : $328\left(\mathrm{M}^{+}+1,100\right), 327\left(\mathrm{M}^{+}, 22\right)$. Anal. Calcd for $\mathrm{C}_{20} \mathrm{H}_{25} \mathrm{NO}_{3}: \mathrm{C}, 73.37 ; \mathrm{H}, 7.70 ; \mathrm{N}, 4.28$. Found: C, 73.26; H, 7.74; N, 4.31 .

(3R,4S)-3-Benzyloxy-4-[(R)-1-hydroxy-2-(1-cyclopentenyl)-ethyl]-1-(3-butenyl)-2azetidinone, (+)-2d. From $87 \mathrm{mg}(0.34 \mathrm{mmol})$ of aldehyde (+)-1d, $70 \mathrm{mg}(61 \%)$ of compound (+)- 
2d was obtained as a colorless solid. Mp: 57-58 ${ }^{\circ} \mathrm{C}$ (hexanes/ethyl acetate). $[\alpha]_{\mathrm{D}}=+55.0$ (c 1.0, $\left.\mathrm{CHCl}_{3}\right) .{ }^{1} \mathrm{H}-\mathrm{NMR}: \delta 1.88(\mathrm{~m}, 2 \mathrm{H}), 2.31(\mathrm{~m}, 9 \mathrm{H}), 3.26(\mathrm{~m}, 1 \mathrm{H}), 3.63(\mathrm{~m}, 1 \mathrm{H}), 3.67(\mathrm{t}, 1 \mathrm{H}, J=5.1 \mathrm{~Hz})$, $4.00(\mathrm{~m}, 1 \mathrm{H}), 4.63(\mathrm{~d}, 1 \mathrm{H}, J=4.9 \mathrm{~Hz}), 4.69$ and $4.97(\mathrm{~d}$, each $1 \mathrm{H}, J=11.7 \mathrm{~Hz}), 5.08(\mathrm{~m}, 2 \mathrm{H}), 5.51(\mathrm{br}$ $\mathrm{s}, 1 \mathrm{H}), 5.76(\mathrm{~m}, 1 \mathrm{H}), 7.35(\mathrm{~m}, 5 \mathrm{H}) .{ }^{13} \mathrm{C}-\mathrm{NMR}: \delta 167.8,140.1,136.9,135.0,128.4,128.0,127.9$, 127.8, 127.6, 80.6, 73.0, 69.0, 60.4, 40.9, 36.0, 34.9, 32.4, 31.8, 23.3. IR $\left(\mathrm{KBr}, \mathrm{cm}^{-1}\right): v 1743 . \mathrm{MS}$ (CI), $m / z: 342\left(\mathrm{M}^{+}+1,100\right), 341\left(\mathrm{M}^{+}, 19\right)$. Anal. Calcd for $\mathrm{C}_{21} \mathrm{H}_{27} \mathrm{NO}_{3}: \mathrm{C}, 73.87 ; \mathrm{H}, 7.97 ; \mathrm{N}$, 4.10. Found: C, 73.97; H, 7.96; N, 4.08.

\section{$(3 R, 4 S)-4-[(R)-1-H y d r o x y-2-(1-c y c l o p e n t e n y l)-e t h y l]-3-m e t h o x y-1-(2-p r o p y n y l)-2-$}

azetidinone, (+)-2e. From $90 \mathrm{mg}(0.54 \mathrm{mmol})$ of aldehyde (+)-1e, $68 \mathrm{mg}(51 \%)$ of compound (+)2e was obtained as a colorless solid. Mp: 87-89 ${ }^{\circ} \mathrm{C}$ (hexanes/ethyl acetate). $[\alpha]_{\mathrm{D}}=+60.0$ (c 1.0, $\left.\mathrm{CHCl}_{3}\right) .{ }^{1} \mathrm{H}-\mathrm{NMR}: \delta 1.88(\mathrm{~m}, 2 \mathrm{H}), 2.35(\mathrm{~m}, 8 \mathrm{H}), 3.60(\mathrm{~s}, 3 \mathrm{H}), 3.81(\mathrm{t}, 1 \mathrm{H}, J=4.9 \mathrm{~Hz}), 3.93(\mathrm{dd}, 1 \mathrm{H}$, $J=17.6,2.7 \mathrm{~Hz}), 4.02(\mathrm{~m}, 1 \mathrm{H}), 4.40(\mathrm{dd}, 1 \mathrm{H}, J=17.6,2.7 \mathrm{~Hz}), 4.48(\mathrm{~d}, 1 \mathrm{H}, J=4.9 \mathrm{~Hz}), 5.55$ (br s, 1H). ${ }^{13}$ C-NMR: $\delta 166.8,139.9,127.2,83.3,72.4,68.5,59.9,59.3,35.8,34.9,32.3,30.8,23.2$. IR $\left(\mathrm{KBr}, \mathrm{cm}^{-1}\right): v$ 3423, 1754. MS (CI), m/z: $250\left(\mathrm{M}^{+}+1,100\right), 249\left(\mathrm{M}^{+}, 15\right)$. Anal. Calcd for $\mathrm{C}_{14} \mathrm{H}_{19} \mathrm{NO}_{3}: \mathrm{C}, 67.45 ; \mathrm{H}, 7.68 ; \mathrm{N}, 5.62$. Found: $\mathrm{C}, 67.34 ; \mathrm{H}, 7.72 ; \mathrm{N}, 5.65$.

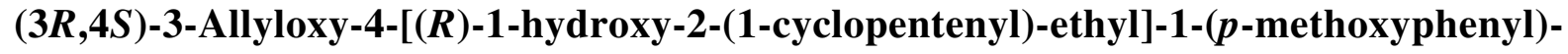
2-azetidinone, (+)-2g. From $100 \mathrm{mg}$ (0.38 mmol) of aldehyde (+)-1g, $109 \mathrm{mg}(83 \%)$ of compound (+)-2g was obtained as a colorless solid. Mp: $67-69^{\circ} \mathrm{C}$ (hexanes/ethyl acetate). $[\alpha]_{\mathrm{D}}=+105.5(c$ 1.2, $\left.\mathrm{CHCl}_{3}\right) .{ }^{1} \mathrm{H}-\mathrm{NMR}: \delta 1.87(\mathrm{~m}, 2 \mathrm{H}), 2.32(\mathrm{~m}, 6 \mathrm{H}), 2.53(\mathrm{~d}, 1 \mathrm{H}, J=2.9 \mathrm{~Hz}), 3.79(\mathrm{~s}, 3 \mathrm{H}), 4.22(\mathrm{~m}$, $1 \mathrm{H}), 4.29(\mathrm{t}, 1 \mathrm{H}, J=5.0 \mathrm{~Hz}), 4.46(\mathrm{ddt}, 1 \mathrm{H}, J=12.7,5.1,1.3 \mathrm{~Hz}), 4.76(\mathrm{~d}, 1 \mathrm{H}, J=5.1 \mathrm{~Hz}), 5.33$ (m, 2H), 5.49 (br s, 1H), $5.96(\mathrm{~m}, 1 \mathrm{H}), 6.86(\mathrm{~m}, 2 \mathrm{H}), 7.43(\mathrm{~m}, 2 \mathrm{H}) .{ }^{13} \mathrm{C}-\mathrm{NMR}: \delta 165.3,156.7,140.3$, $133.2,130.9,127.1,120.4,118.2,114.1,80.4,72.5,69.3,60.5,55.4,35.6,35.0,32.5,23.3$. IR (KBr, $\left.\mathrm{cm}^{-1}\right): \vee 3522,1745$. MS (EI), $m / z: 344\left(\mathrm{M}^{+}+1,9\right), 343\left(\mathrm{M}^{+}, 100\right)$. Anal. Calcd for $\mathrm{C}_{20} \mathrm{H}_{25} \mathrm{NO}_{4}$ : C, 69.95; H, 7.34; N, 4.08. Found: C, 70.05; H, 7.37; N, 4.10.

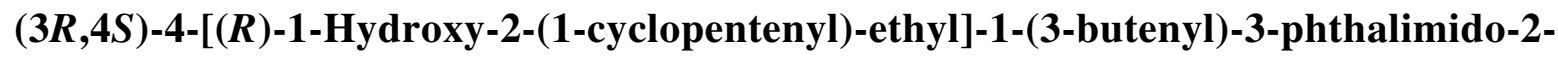
azetidinone, (-)-2i. From $186 \mathrm{mg}(0.62 \mathrm{mmol})$ of aldehyde (-)-1i, $121 \mathrm{mg}(51 \%)$ of compound (-)2i was obtained as a colorless oil. $[\alpha]_{\mathrm{D}}=-22.1\left(c\right.$ 1.0, $\left.\mathrm{CHCl}_{3}\right) .{ }^{1} \mathrm{H}-\mathrm{NMR}: \delta 1.69(\mathrm{~m}, 2 \mathrm{H}), 1.95(\mathrm{~m}$, 2H), $2.15(\mathrm{~m}, 4 \mathrm{H}), 2.26(\mathrm{~d}, 1 \mathrm{H}, J=3.2 \mathrm{~Hz}), 2.47(\mathrm{~m}, 2 \mathrm{H}), 3.46(\mathrm{~m}, 1 \mathrm{H}), 3.69(\mathrm{~m}, 1 \mathrm{H}), 3.84(\mathrm{dd}, 1 \mathrm{H}, J$ $=8.8,5.1 \mathrm{~Hz}), 3.99(\mathrm{tt}, 1 \mathrm{H}, J=8.8,2.9 \mathrm{~Hz}), 5.15(\mathrm{~m}, 2 \mathrm{H}), 5.33(\mathrm{~d}, 1 \mathrm{H}, J=5.1 \mathrm{~Hz}), 5.37(\mathrm{br} \mathrm{s}, 1 \mathrm{H})$, 
$5.85(\mathrm{~m}, 1 \mathrm{H}), 7.78(\mathrm{~m}, 2 \mathrm{H}), 7.86(\mathrm{~m}, 2 \mathrm{H}) .{ }^{13} \mathrm{C}-\mathrm{NMR}: \delta 167.4,163.8,138.8,135.2,134.6,131.4$, 128.5, 123.7, 117.1, 69.1, 61.3, 54.7, 41.7, 36.4, 34.7, 32.4, 31.9, 23.1. IR $\left(\mathrm{CHCl}_{3}, \mathrm{~cm}^{-1}\right): v 3402$, 1757. MS (CI), $m / z: 381\left(\mathrm{M}^{+}+1,100\right), 380\left(\mathrm{M}^{+}, 11\right)$. Anal. Calcd for $\mathrm{C}_{22} \mathrm{H}_{24} \mathrm{~N}_{2} \mathrm{O}_{4}: \mathrm{C}, 69.46 ; \mathrm{H}$, 6.36; N, 7.36. Found: C, 69.38; H, 6.33; N, 7.40.

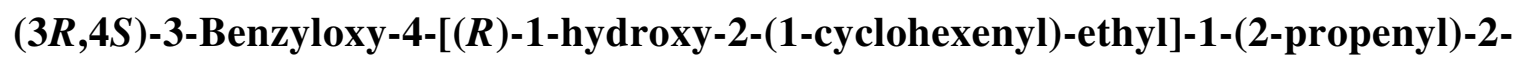
azetidinone, (+)-3b. From $100 \mathrm{mg}(0.41 \mathrm{mmol})$ of aldehyde (+)-1c, $51 \mathrm{mg}(37 \%)$ of compound (+)-3b was obtained as a colorless solid. Mp: 76-78 ${ }^{\circ} \mathrm{C}$ (hexanes/ethyl acetate). $[\alpha]_{\mathrm{D}}=+31.6(c$ 1.0, $\left.\mathrm{CHCl}_{3}\right) .{ }^{1} \mathrm{H}-\mathrm{NMR}: \delta 1.58(\mathrm{~m}, 5 \mathrm{H}), 1.96(\mathrm{~m}, 4 \mathrm{H}), 2.29(\mathrm{~m}, 2 \mathrm{H}), 3.66(\mathrm{t}, 1 \mathrm{H}, J=5.4 \mathrm{~Hz}), 3.83$ (ddt, $1 \mathrm{H}, J=15.7,6.8,1.2 \mathrm{~Hz}), 3.95(\mathrm{~m}, 1 \mathrm{H}), 4.17(\mathrm{ddt}, 1 \mathrm{H}, J=15.7,5.1,1.6 \mathrm{~Hz}), 4.66(\mathrm{~d}, 1 \mathrm{H}, J=5.1 \mathrm{~Hz})$, 4.70 and $4.99(\mathrm{~d}$, each 1H, $J=11.7 \mathrm{~Hz}), 5.23(\mathrm{~m}, 2 \mathrm{H}), 5.52(\mathrm{br} \mathrm{s}, 1 \mathrm{H}), 5.77(\mathrm{~m}, 1 \mathrm{H}), 7.35(\mathrm{~m}, 5 \mathrm{H})$. ${ }^{13}$ C-NMR: $\delta 167.8,137.0,133.9,132.2,128.6,128.2,128.0,125.4,118.4,80.9,73.3,68.8,60.8,44.3$, 42.9, 28.3, 25.4, 23.0, 22.4. IR (KBr, cm-1): v 3443, 1726. MS (CI), $m / z: 342\left(\mathrm{M}^{+}+1,100\right), 341$ $\left(\mathrm{M}^{+}, 15\right)$. Anal. Calcd for $\mathrm{C}_{21} \mathrm{H}_{27} \mathrm{NO}_{3}$ : C, 73.87; H, 7.97; N, 4.10. Found: C, 73.96; H, 7.92; N, 4.12.

\section{$(3 R, 4 S)-4-[(R)-1-H y d r o x y-3-p h e n y l-3-b u t e n y l]-3-m e t h o x y-1-(p-m e t h o x y-p h e n y l)-2-$}

azetidinone, (+)-4a. From $100 \mathrm{mg}(0.43 \mathrm{mmol})$ of aldehyde (+)-1a, $100 \mathrm{mg}(66 \%)$ of compound (+)-4a was obtained as a colorless oil. $[\alpha]_{\mathrm{D}}=+68.9\left(c\right.$ 1.0, $\left.\mathrm{CHCl}_{3}\right) .{ }^{1} \mathrm{H}-\mathrm{NMR}: \delta 2.35(\mathrm{~d}, 1 \mathrm{H}, J=4.0$ Hz), $2.63(\mathrm{dd}, 1 \mathrm{H}, J=10.3,9.6 \mathrm{~Hz}), 2.97(\mathrm{ddd}, 1 \mathrm{H}, J=14.1,3.7,1.0 \mathrm{~Hz}), 3.69$ (s, 3H), 3.79 (s, 3H), $4.12(\mathrm{~m}, 1 \mathrm{H}), 4.30(\mathrm{t}, 1 \mathrm{H}, J=5.5 \mathrm{~Hz}), 4.62(\mathrm{~d}, 1 \mathrm{H}, J=5.4 \mathrm{~Hz}), 5.16(\mathrm{br} \mathrm{s}, 1 \mathrm{H}), 5.44(\mathrm{~d}, 1 \mathrm{H}, J=1.0$ $\mathrm{Hz}), 6.86$ (m, 2H), 7.30 (m, 2H), 7.39 (m, 5H). ${ }^{13} \mathrm{C}-\mathrm{NMR}: \delta 165.4,157.0,144.6,140.1,130.9,128.6$, 128.0, 126.4, 120.7, 115.8, 114.3, 82.8, 69.7, 60.7, 60.0, 55.7, 39.7. IR $\left(\mathrm{CHCl}_{3}, \mathrm{~cm}^{-1}\right): v 3475,1747$. MS (CI), m/z : $354\left(\mathrm{M}^{+}, 100\right), 353$ (11). Anal. Calcd for $\mathrm{C}_{21} \mathrm{H}_{23} \mathrm{NO}_{4}$ : C, 71.37; H, 6.56; N, 3.96. Found: C, 71.29; H, 6.52; N, 3.99.

\section{$(3 R, 4 S)-3-B e n z y l o x y-4-[(R)-1-h y d r o x y-3-p h e n y l-3-b u t e n y l]-1-(3-b u t e n y l)-2-$}

azetidinone, (+)-4b. From $75 \mathrm{mg}(0.29 \mathrm{mmol})$ of aldehyde (+)-1d, $65 \mathrm{mg}(60 \%)$ of compound (+)4b was obtained as a colorless oil. $[\alpha]_{\mathrm{D}}=+4.6\left(c 0.8, \mathrm{CHCl}_{3}\right) .{ }^{1} \mathrm{H}-\mathrm{NMR}: \delta 2.21(\mathrm{~d}, 1 \mathrm{H}, J=2.4 \mathrm{~Hz})$, $2.37(\mathrm{~m}, 2 \mathrm{H}), 2.47(\mathrm{dd}, 1 \mathrm{H}, J=13.9,9.8 \mathrm{~Hz}), 3.08(\mathrm{ddd}, 1 \mathrm{H}, J=13.9,3.4,1.1 \mathrm{~Hz}), 3.27(\mathrm{~m}, 1 \mathrm{H}), 3.57$ $(\mathrm{m}, 1 \mathrm{H}), 3.67(\mathrm{dd}, 1 \mathrm{H}, J=7.1,5.1 \mathrm{~Hz}), 3.94(\mathrm{~m}, 1 \mathrm{H}), 4.64(\mathrm{~d}, 1 \mathrm{H}, J=4.9 \mathrm{~Hz}), 4.68$ and $5.0(\mathrm{~d}$, each $1 \mathrm{H}, J=11.2 \mathrm{~Hz}), 5.08(\mathrm{~m}, 2 \mathrm{H}), 5.18(\mathrm{br} \mathrm{s}, 1 \mathrm{H}), 5.50(\mathrm{br} \mathrm{s}, 1 \mathrm{H}), 5.76(\mathrm{~m}, 1 \mathrm{H}), 7.22(\mathrm{~m}, 5 \mathrm{H}), 7.38(\mathrm{~m}$, 
5H). ${ }^{13} \mathrm{C}-\mathrm{NMR}: \delta 167.8,143.9,139.1,136.8,135.0,128.5,128.4,128.3,128.1,127.8,126.1,116.9$, 115.8, 80.5, 73.2, 69.6, 60.6, 40.8, 40.0, 31.8. IR $\left(\mathrm{CHCl}_{3}, \mathrm{~cm}^{-1}\right): v 1743 . \mathrm{MS}(\mathrm{EI}), \mathrm{m} / z: 378\left(\mathrm{M}^{+}+1\right.$, 7), $377\left(\mathrm{M}^{+}, 100\right)$. Anal. Calcd for $\mathrm{C}_{24} \mathrm{H}_{27} \mathrm{NO}_{3}$ : C, 76.36; H, 7.21; N, 3.71. Found: C, 76.46; H, $7.25 ; \mathrm{N}, 3.69$.

$(3 R, 4 S)-4-[(R)-1-H y d r o x y-3-p h e n y l-3-b u t e n y l]-3-m e t h o x y-1-(2-p r o p y n y l)-2-$

azetidinone, (+)-4c. From $100 \mathrm{mg}(0.60 \mathrm{mmol})$ of aldehyde (+)-1e, $110 \mathrm{mg}(64 \%)$ of compound (+)-4c was obtained as a colorless oil. $[\alpha]_{\mathrm{D}}=+16.3\left(c\right.$ 1.0, $\left.\mathrm{CHCl}_{3}\right) .{ }^{1} \mathrm{H}-\mathrm{NMR}: \delta 2.30(\mathrm{t}, 1 \mathrm{H}, J=2.5$ Hz), $2.46(\mathrm{~d}, 1 \mathrm{H}, J=3.4 \mathrm{~Hz}), 2.62(\mathrm{dd}, 1 \mathrm{H}, J=13.8,9.1 \mathrm{~Hz}), 2.99$ (ddd, 1H, $J=14.1,3.9,0.8 \mathrm{~Hz})$, 3.59 (s, 3H), $3.82(\mathrm{t}, 1 \mathrm{H}, J=5.4 \mathrm{~Hz}), 3.95(\mathrm{~m}, 1 \mathrm{H}), 3.96$ and $4.36(\mathrm{dd}$, each $1 \mathrm{H}, J=17.6,2.5 \mathrm{~Hz})$, $4.45(\mathrm{~d}, 1 \mathrm{H}, J=5.0 \mathrm{~Hz}), 5.25(\mathrm{~d}, 1 \mathrm{H}, J=1.0 \mathrm{~Hz}), 5.49(\mathrm{~d}, 1 \mathrm{H}, J=1.3 \mathrm{~Hz}), 7.31(\mathrm{~m}, 3 \mathrm{H}), 7.70(\mathrm{~m}$, 2H). ${ }^{13}$ C-NMR: $\delta$ 166.8, 143.8, 139.6, 128.3, 127.8, 126.0, 115.7, 83.1, 72.5, 69.0, 60.0, 59.2, 43.1, 39.9, 30.8. IR $\left(\mathrm{CHCl}_{3}, \mathrm{~cm}^{-1}\right): v$ 3462, 1755. MS (CI), m/z: $286\left(\mathrm{M}^{+}+1,100\right), 285\left(\mathrm{M}^{+}, 5\right)$. Anal. Calcd for $\mathrm{C}_{17} \mathrm{H}_{19} \mathrm{NO}_{3}$ : C, 71.56; H, 6.71; N, 4.91. Found: C, 71.48; H, 6.68; N, 4.94.

$(3 R, 4 S)-3-A l l y l o x y-4-[(R)-1-h y d r o x y-3-p h e n y l-3-b u t e n y l]-1-(p-m e t h o x y-p h e n y l)-2-$

azetidinone, (+)-4e. From $82 \mathrm{mg}(0.31 \mathrm{mmol})$ of aldehyde (+)-1g, $50 \mathrm{mg}(42 \%)$ of compound (+)4e was obtained as a colorless oil. $[\alpha]_{\mathrm{D}}=+35.5\left(c\right.$ 1.1, $\left.\mathrm{CHCl}_{3}\right) .{ }^{1} \mathrm{H}-\mathrm{NMR}: \delta 2.38(\mathrm{~d}, 1 \mathrm{H}, J=3.7$ Hz), $2.62(\mathrm{dd}, 1 \mathrm{H}, J=14.2,9.5 \mathrm{~Hz}), 3.01(\mathrm{dd}, 1 \mathrm{H}, J=14.2,3.7 \mathrm{~Hz}), 3.78(\mathrm{~s}, 3 \mathrm{H}), 4.18(\mathrm{~m}, 2 \mathrm{H}), 4.28$ (t, $1 \mathrm{H}, J=5.5 \mathrm{~Hz}), 4.47(\mathrm{ddt}, 1 \mathrm{H}, J=12.3,5.5,1.2 \mathrm{~Hz}), 4.75(\mathrm{~d}, 1 \mathrm{H}, J=5.1 \mathrm{~Hz}), 5.16$ (br s, 1H), 5.34 $(\mathrm{m}, 2 \mathrm{H}), 5.44$ (br s, 1H), $5.97(\mathrm{~m}, 1 \mathrm{H}), 6.84(\mathrm{~m}, 2 \mathrm{H}), 7.43(\mathrm{~m}, 7 \mathrm{H}) .{ }^{13} \mathrm{C}-\mathrm{NMR}: \delta$ 165.2, 156.7, 144.3, 139.7, 133.2, 130.7, 128.3, 127.7, 126.2, 120.4, 118.7, 115.6, 114.0, 80.4, 72.6, 69.6, 60.7, 55.4, 39.5. IR $\left(\mathrm{KBr}, \mathrm{cm}^{-1}\right): v$ 3528, 1742. MS (CI), m/z: $380\left(\mathrm{M}^{+}+1,100\right), 379\left(\mathrm{M}^{+}, 11\right)$. Anal. Calcd for $\mathrm{C}_{23} \mathrm{H}_{25} \mathrm{NO}_{4}$ : C, 72.80; H, 6.64; N, 3.69. Found: C, 72.91; H, 6.60; N, 3.66.

(3R,4S)-4-[(R)-1-Hydroxy-3-phenyl-3-butenyl]-1-(3-butenyl)-3-phthalimido-2-

azetidinone, (-)-4g. From $77 \mathrm{mg}(0.26 \mathrm{mmol})$ of aldehyde (-)-1i, $50 \mathrm{mg}(46 \%)$ of compound (-)4g was obtained as a colorless oil. $[\alpha]_{\mathrm{D}}=-41.0\left(c\right.$ 1.8, $\left.\mathrm{CHCl}_{3}\right) .{ }^{1} \mathrm{H}-\mathrm{NMR}: \delta 2.15$ (br s, $\left.1 \mathrm{H}\right), 2.41(\mathrm{~m}$, 4H), $3.45(\mathrm{~m}, 1 \mathrm{H}), 3.67(\mathrm{~m}, 1 \mathrm{H}), 3.85(\mathrm{~m}, 2 \mathrm{H}), 5.14(\mathrm{~m}, 2 \mathrm{H}), 5.29(\mathrm{~s}, 1 \mathrm{H}), 5.33(\mathrm{~d}, 1 \mathrm{H}, J=5.1 \mathrm{~Hz})$, $6.96(\mathrm{~m}, 5 \mathrm{H}), 7.83(\mathrm{~m}, 4 \mathrm{H}) .{ }^{13} \mathrm{C}-\mathrm{NMR}: \delta 167.3,163.7,143.7,139.7,135.2,134.6,131.6,128.2$, 127.7, 125.8, 123.9, 117.1, 116.8, 68.9, 61.3, 54.9, 41.8, 41.2, 31.8. IR $\left(\mathrm{CHCl}_{3}, \mathrm{~cm}^{-1}\right): v 3424,1751$. 
MS (CI), m/z: $417\left(\mathrm{M}^{+}+1,100\right), 416\left(\mathrm{M}^{+}, 7\right)$. Anal. Calcd for $\mathrm{C}_{25} \mathrm{H}_{24} \mathrm{~N}_{2} \mathrm{O}_{4}: \mathrm{C}, 72.10 ; \mathrm{H}, 5.81 ; \mathrm{N}$, 6.73. Found: C, 72.02; H, 5.79; N, 6.77.

\section{General Procedure for the Preparation of the $O$-Acetylmandelates of Homoallylic} Alcohol (+)-2b. $\quad(R)-\quad$ or $\quad(S)-O$-Acetylmandelic acid $(20 \quad \mathrm{mg}, \quad 0.10 \quad \mathrm{mmol})$ and $4-$ dimethylaminopyridine (DMAP) (cat.) were added to a solution of the homoallylic alcohol (+)-2b $(0.09 \mathrm{mmol})$ in dichloromethane $(1.0 \mathrm{~mL})$ followed by a solution of dicyclohexylcarbodiimide (DCC) $(37 \mathrm{mg}, 0.18 \mathrm{mmol})$ in dichloromethane $(500 \mu \mathrm{L})$ at $0{ }^{\circ} \mathrm{C}$. The reaction mixture was allowed to warm to room temperature and stirred for $16 \mathrm{~h}$. The solvent was removed under reduced pressure and diethyl ether was added. The mixture was then filtered and the filtrate was concentrated under reduced pressure. Chromatography of the residue eluting with hexanes/ethyl acetate mixtures gave analytically pure $O$-acetylmandelates.

$(R)$ - $O$-Acetylmandelate $\quad$ of $\quad(3 R, 4 S)-4-[(R)-1$-hydroxy-2-(1-cyclopentenyl)]-1-(pmethoxyphenyl)-3-phenoxy-2-azetidinone. From $30 \mathrm{mg}(0.08 \mathrm{mmol})$ of alcohol (+)-2b, $33 \mathrm{mg}$ $(75 \%)$ of its $(R)-O$-acetylmandelate was obtained as a colorless oil. $[\alpha]_{\mathrm{D}}=+79.6\left(c 1.4, \mathrm{CHCl}_{3}\right) .{ }^{1} \mathrm{H}-$ NMR: $\delta 1.61(\mathrm{~m}, 2 \mathrm{H}), 1.83(\mathrm{~m}, 2 \mathrm{H}), 2.13(\mathrm{~s}, 3 \mathrm{H}), 2.27(\mathrm{~m}, 2 \mathrm{H}), 2.58(\mathrm{~m}, 2 \mathrm{H}), 3.82(\mathrm{~s}, 3 \mathrm{H}), 4.53(\mathrm{dd}$, $1 \mathrm{H}, J=5.1,4.4 \mathrm{~Hz}), 5.22(\mathrm{~d}, 1 \mathrm{H}, J=5.4 \mathrm{~Hz}), 5.44(\mathrm{br} \mathrm{s}, 1 \mathrm{H}), 5.58(\mathrm{dt}, 1 \mathrm{H}, J=6.1,4.1 \mathrm{~Hz}), 5.83(\mathrm{~s}$, 1H), 7.13 (m, 14H). ${ }^{13} \mathrm{C}-\mathrm{NMR}: \delta$ 169.8, 167.9, 163.2, 157.4, 157.0, 138.7, 130.2, 129.6, 129.1, 128.7, $128.4,127.8,127.6,122.5,120.3,115.9,114.5,79.5,74.6,71.0,57.6,55.6,35.1,33.6,32.6,23.6$, 20.8. IR $\left(\mathrm{CHCl}_{3}, \mathrm{~cm}^{-1}\right): v 1743,1648$. MS (CI), $m / z: 556\left(\mathrm{M}^{+}+1,100\right), 555\left(\mathrm{M}^{+}, 8\right)$. Anal. Calcd for $\mathrm{C}_{33} \mathrm{H}_{33} \mathrm{NO}_{7}$ : C, 71.23; H, 5.99; N, 2.52. Found: C, 71.34; H, 5.96; N, 2.50 .

$(S)$-O-Acetylmandelate of $\quad(3 R, 4 S)-4-[(R)-1$-hydroxy-2-(1-cyclopentenyl)]-1-(pmethoxyphenyl)-3-phenoxy-2-azetidinone. From $30 \mathrm{mg}(0.08 \mathrm{mmol})$ of alcohol (+)-2b, $31 \mathrm{mg}$ $(71 \%)$ of its $(S)-O$-acetylmandelate was obtained as a colorless solid. Mp: $125-127^{\circ} \mathrm{C}$ (hexanes/ethyl acetate). $[\alpha]_{\mathrm{D}}=+100.2\left(\right.$ ( $\left.2.0, \mathrm{CHCl}_{3}\right) .{ }^{1} \mathrm{H}-\mathrm{NMR}: \delta 1.22(\mathrm{~m}, 1 \mathrm{H}), 1.61(\mathrm{~m}, 3 \mathrm{H}), 1.98(\mathrm{~m}, 2 \mathrm{H}), 2.11$ (s, 3H), $2.45(\mathrm{~m}, 2 \mathrm{H}), 3.82(\mathrm{~s}, 3 \mathrm{H}), 4.60(\mathrm{t}, 1 \mathrm{H}, J=5.6 \mathrm{~Hz}), 4.87$ (br s, $1 \mathrm{H}), 5.45$ (d, $1 \mathrm{H}, J=4.9 \mathrm{~Hz})$, $5.50(\mathrm{~s}, 1 \mathrm{H}), 5.66(\mathrm{~m}, 1 \mathrm{H}), 7.17(\mathrm{~m}, 14 \mathrm{H}) .{ }^{13} \mathrm{C}-\mathrm{NMR}: \delta$ 169.8, 167.8, 163.0, 157.4, 157.0, 138.1, 133.7, 130.0, 129.6, 129.0, 128.5, 127.8, 127.7, 122.5, 119.9, 115.6, 114.4, 79.2, 74.1, 71.4, 57.9, 55.5, 34.5, 34.0, 32.3, 23.2, 20.6. IR $\left(\mathrm{CHCl}_{3}, \mathrm{~cm}^{-1}\right): v$ 1745, 1649. MS (CI), m/z: $556\left(\mathrm{M}^{+}+1,100\right), 555$ 
$\left(\mathrm{M}^{+}, 11\right)$. Anal. Calcd for $\mathrm{C}_{33} \mathrm{H}_{33} \mathrm{NO}_{7}: \mathrm{C}, 71.23 ; \mathrm{H}, 5.99 ; \mathrm{N}, 2.52$. Found: $\mathrm{C}, 71.33 ; \mathrm{H}, 6.03 ; \mathrm{N}$, 2.55

Methanesulfonate of $(3 R, 4 S)-3$-propargyloxy-4-[(R)-1-hydroxy-2-(1-cyclo-pentenyl)ethyl]-1-(p-methoxyphenyl)-2-azetidinone, (+)-5b. From $224 \mathrm{mg}(0.66 \mathrm{mmol})$ of alcohol (+)$\mathbf{2 h}, 205 \mathrm{mg}(74 \%)$ of compound (+)-5b was obtained as a colorless oil. $[\alpha]_{\mathrm{D}}=+77.2$ (c 2.4, $\left.\mathrm{CHCl}_{3}\right) .{ }^{1} \mathrm{H}-\mathrm{NMR}: \delta 1.90(\mathrm{~m}, 2 \mathrm{H}), 2.33(\mathrm{~m}, 4 \mathrm{H}), 2.57(\mathrm{~m}, 4 \mathrm{H}), 2.71(\mathrm{~m}, 2 \mathrm{H}), 3.79(\mathrm{~s}, 3 \mathrm{H}), 4.51(\mathrm{~m}$, 3H), $5.00(\mathrm{~d}, 1 \mathrm{H}, J=5.4 \mathrm{~Hz}), 5.15(\mathrm{ddd}, 1 \mathrm{H}, J=7.6,5.9,4.9 \mathrm{~Hz}), 5.30$ (s, 1H), 5.59 (br s, 1H), 6.88 (m, 2H), 7.35 (m, 2H). ${ }^{13} \mathrm{C}-\mathrm{NMR}: \delta 164.3,156.9,138.1,130.2,129.4,119.8,114.3,80.2,79.4,78.2$, 76.2, 58.8, 57.7, 55.4, 38.1, 35.6, 34.1, 32.5, 23.5. IR $\left(\mathrm{CHCl}_{3}, \mathrm{~cm}^{-1}\right): v 1744 . \mathrm{MS}(\mathrm{CI}), \mathrm{m} / \mathrm{z}: 420\left(\mathrm{M}^{+}\right.$ $+1,100), 419\left(\mathrm{M}^{+}, 9\right)$. Anal. Calcd for $\mathrm{C}_{21} \mathrm{H}_{25} \mathrm{NSO}_{6}$ : C, 60.13; H, 6.01; N, 3.34. Found: C, 60.23; H, 6.04; N, 3.32.

Methanesulfonate of $(3 R, 4 S)-4-[(R)-1-h y d r o x y-3-p h e n y l-3-b u t e n y l]-3-m e t h o x y-1-(3-$ butynyl)-2-azetidinone, (+)-5c. From $140 \mathrm{mg}(0.47 \mathrm{mmol})$ of alcohol (+)-4d, $199 \mathrm{mg}(70 \%)$ of compound (+)-5c was obtained as a colorless oil. $[\alpha]_{\mathrm{D}}=+3.9\left(\right.$ c 1.7, $\left.\mathrm{CHCl}_{3}\right) .{ }^{1} \mathrm{H}-\mathrm{NMR}: \delta 1.96(\mathrm{t}$, $1 \mathrm{H}, J=2.6 \mathrm{~Hz}), 2.45(\mathrm{~m}, 2 \mathrm{H}), 2.85(\mathrm{~s}, 3 \mathrm{H}), 3.00(\mathrm{~m}, 2 \mathrm{H}), 3.27(\mathrm{~m}, 1 \mathrm{H}), 3.57(\mathrm{~s}, 3 \mathrm{H}), 3.72(\mathrm{~m}, 1 \mathrm{H})$, $4.01(\mathrm{dd}, 1 \mathrm{H}, J=7.8,5.1 \mathrm{~Hz}), 4.43(\mathrm{~d}, 1 \mathrm{H}, J=5.1 \mathrm{~Hz}), 5.03(\mathrm{td}, 1 \mathrm{H}, J=7.8,4.1 \mathrm{~Hz}), 5.27(\mathrm{br} \mathrm{s}, 1 \mathrm{H})$, $5.53(\mathrm{~d}, 1 \mathrm{H}, J=1.0 \mathrm{~Hz}), 7.39(\mathrm{~m}, 5 \mathrm{H}) .{ }^{13} \mathrm{C}-\mathrm{NMR}: \delta 167.6,142.7,139.5,128.6,128.1,126.1,117.4$, 82.7, 80.8, 70.4, 59.0, 58.5, 39.7, 38.9, 38.0, 31.5, 17.1. IR $\left(\mathrm{CHCl}_{3}, \mathrm{~cm}^{-1}\right): v 1746 . \mathrm{MS}(\mathrm{CI}), m / z: 378$ $\left(\mathrm{M}^{+}+1,100\right), 377\left(\mathrm{M}^{+}, 11\right)$. Anal. Calcd for $\mathrm{C}_{19} \mathrm{H}_{23} \mathrm{NSO}_{5}$ : C, 60.46; H, 6.14; N, 3.71. Found: C, $60.58 ; \mathrm{H}, 6.10 ; \mathrm{N}, 3.68$.

Cycloadduct (+)-6c. From $36 \mathrm{mg}(0.10 \mathrm{mmol})$ of methanesulfonate (+)-5c and after heating at $140{ }^{\circ} \mathrm{C}$ for $44 \mathrm{~h}, 15 \mathrm{mg}(56 \%)$ of compound (+)-6c was obtained as a colorless oil. $[\alpha]_{\mathrm{D}}=+174.8$ $\left(c\right.$ 0.5, $\left.\mathrm{CHCl}_{3}\right) .{ }^{1} \mathrm{H}-\mathrm{NMR}: \delta 2.27(\mathrm{~m}, 2 \mathrm{H}), 2.79$ (dddd, $\left.1 \mathrm{H}, J=13.0,10.0,6.7,1.4 \mathrm{~Hz}\right), 3.15(\mathrm{~m}, 3 \mathrm{H})$, $3.32(\mathrm{dd}, 1 \mathrm{H}, J=9.5,4.1 \mathrm{~Hz}), 3.66(\mathrm{~s}, 3 \mathrm{H}), 3.96(\mathrm{ddd}, 1 \mathrm{H}, J=13.0,5.2,2.2 \mathrm{~Hz}), 4.58(\mathrm{dd}, 1 \mathrm{H}, J=$ 4.1, $1.4 \mathrm{~Hz}), 5.79(\mathrm{~d}, 1 \mathrm{H}, J=1.1 \mathrm{~Hz}), 5.97(\mathrm{t}, 1 \mathrm{H}, J=1.6 \mathrm{~Hz}), 7.36(\mathrm{~m}, 5 \mathrm{H}) .{ }^{13} \mathrm{C}-\mathrm{NMR}: \delta 167.2$, $140.6,135.1,131.4,128.3,127.4,125.1,121.1,121.0,85.1,59.4,59.3,39.6,38.0,33.4,28.7$. IR 
$\left(\mathrm{CHCl}_{3}, \mathrm{~cm}^{-1}\right): v$ 1751. MS (ES), m/z: $304\left(\mathrm{M}^{+}+23,14\right), 282\left(\mathrm{M}^{+}+1,100\right), 281\left(\mathrm{M}^{+}, 7\right)$. Anal. Calcd for $\mathrm{C}_{18} \mathrm{H}_{19} \mathrm{NO}_{2}$ : C, 76.84; H, 6.81; N, 4.98. Found: C, 76.95; H, 6.85; N, 4.94.

Tricyclic $\boldsymbol{\beta}$-Lactam (+)-7a. From $15 \mathrm{mg}(0.06 \mathrm{mmol})$ of cycloadduct (+)-6a, $100 \mathrm{mg}(100 \%)$ of tricycle (+)-7a was obtained as a colorless oil. $[\alpha]_{\mathrm{D}}=+13.2\left(c 0.4, \mathrm{CHCl}_{3}\right) .{ }^{1} \mathrm{H}-\mathrm{NMR}: \delta 2.09(\mathrm{~m}$, 2H), $2.89(\mathrm{~m}, 7 \mathrm{H}), 3.51(\mathrm{~s}, 3 \mathrm{H}), 4.05(\mathrm{~m}, 1 \mathrm{H}), 4.69(\mathrm{~d}, 1 \mathrm{H}, J=4.5 \mathrm{~Hz}), 4.79(\mathrm{~d}, 1 \mathrm{H}, J=4.5 \mathrm{~Hz}), 7.00$ (s, 1H), 7.04 (s, 1H). IR $\left(\mathrm{CHCl}_{3}, \mathrm{~cm}^{-1}\right): v$ 1762. MS (CI), m/z: $244\left(\mathrm{M}^{+}+1,100\right), 243\left(\mathrm{M}^{+}, 16\right)$. Anal. Calcd for $\mathrm{C}_{15} \mathrm{H}_{17} \mathrm{NO}_{2}$ : C, 74.05; H, 7.04; N, 5.76. Found: C, 73.97; H, 7.00; N, 5.79.

Bicyclic Amide (土)-8b. From $50 \mathrm{mg}(0.11 \mathrm{mmol})$ of methanesulfonate (+)-5d, $27 \mathrm{mg}(69 \%)$ of bicycle ( \pm )-8b was obtained as a colorless solid. Mp: 186-187 ${ }^{\circ} \mathrm{C}$ (hexanes/ethyl acetate). ${ }^{1} \mathrm{H}-$ NMR: $\delta 3.08(\mathrm{dd}, 1 \mathrm{H}, J=11.2,16.3 \mathrm{~Hz}), 3.38(\mathrm{dd}, 1 \mathrm{H}, J=3.8,16.3 \mathrm{~Hz}), 3.82(\mathrm{~s}, 3 \mathrm{H}), 4.36(\mathrm{dd}, 1 \mathrm{H}$, $J=3.8,11.2 \mathrm{~Hz}), 4.99(\mathrm{~d}, 1 \mathrm{H}, J=15.1 \mathrm{~Hz}), 5.10(\mathrm{~d}, 1 \mathrm{H}, J=14.9 \mathrm{~Hz}), 6.90(\mathrm{~m}, 2 \mathrm{H}), 7.13(\mathrm{~d}, 1 \mathrm{H}, J=$ 8.1 Hz), 7.47 (m, 9H), 8.38 (br s, 1H). ${ }^{13} \mathrm{C}-\mathrm{NMR}: \delta$ 168.8, 156.6, 140.6, 140.2, 132.8, 132.6, 130.4, $128.8,127.7,127.4,127.0,125.4,124.6,121.5,114.2,74.6,68.3,55.5,31.3 . \mathrm{IR}\left(\mathrm{KBr}, \mathrm{cm}^{-1}\right): v 3318$, 1665. MS (CI), $m / z: 360\left(\mathrm{M}^{+}+1,100\right), 359\left(\mathrm{M}^{+}, 7\right)$. Anal. Calcd for $\mathrm{C}_{23} \mathrm{H}_{21} \mathrm{NO}_{3}: \mathrm{C}, 76.86 ; \mathrm{H}$, 5.89; N, 3.90. Found: C, 76.97; H, 5.92; N, 3.88.

Bicyclic $\boldsymbol{\beta}$-Lactam (+)-11b. From $60 \mathrm{mg}(0.20 \mathrm{mmol})$ of ene adduct (+)-4d, $47 \mathrm{mg}(36 \%)$ of compound (+)-11b was obtained as a colorless oil. $[\alpha]_{\mathrm{D}}=+107.1\left(\right.$ c $\left.1.8, \mathrm{CHCl}_{3}\right) .{ }^{1} \mathrm{H}-\mathrm{NMR}: \delta 2.23$ (m, 2H), $2.87(\mathrm{~m}, 3 \mathrm{H}), 3.34(\mathrm{~d}, 1 \mathrm{H}, J=4.9 \mathrm{~Hz}), 3.41(\mathrm{~s}, 3 \mathrm{H}), 3.52(\mathrm{~m}, 2 \mathrm{H}), 3.72(\mathrm{~d}, 1 \mathrm{H}, J=4.6 \mathrm{~Hz})$, $3.80(\mathrm{~m}, 1 \mathrm{H}), 3.97(\mathrm{~m}, 1 \mathrm{H}), 6.19(\mathrm{~s}, 1 \mathrm{H}), 7.43(\mathrm{~m}, 20 \mathrm{H}) .{ }^{13} \mathrm{C}-\mathrm{NMR}: \delta$ 167.4, 158.8, 148.9, 138.4, $137.3,136.9,136.6,129.3,129.2,129.1,128.8,128.6,128.5,128.3,126.9,126.0,84.1,67.6,62.4$, 59.5, 50.5, 40.7, 39.3, 39.1, 37.8. IR $\left(\mathrm{CHCl}_{3}, \mathrm{~cm}^{-1}\right): v$ 1743. $\mathrm{MS}(\mathrm{EI}), \mathrm{m} / z: 650\left(\mathrm{M}^{+}, 6\right), 574\left(\mathrm{M}^{+}{ }_{-}\right.$ 76, 100). Anal. Calcd for $\mathrm{C}_{36} \mathrm{H}_{37} \mathrm{NO}_{3} \mathrm{Sn}: \mathrm{C}, 66.48 ; \mathrm{H}, 5.73 ; \mathrm{N}, 2.15$. Found: C, 66.62; H, 5.77; N, 2.11 . 